\title{
DISCRETENESS CRITERIA FOR ISOMETRIC GROUPS ACTING ON COMPLEX HYPERBOLIC SPACES
}

\author{
XI FU \\ (Received 5 August 2009)
}

\begin{abstract}
In this paper, four new discreteness criteria for isometric groups on complex hyperbolic spaces are proved, one of which shows that the Condition $\mathrm{C}$ hypothesis in Cao ['Discrete and dense subgroups acting on complex hyperbolic space', Bull. Aust. Math. Soc. 78 (2008), 211-224, Theorem 1.4] is removable; another shows that the parabolic condition hypothesis in Li and Wang ['Discreteness criteria for Möbius groups acting on $\overline{\mathbb{R}}^{n}$ II', Bull. Aust. Math. Soc. 80 (2009), 275-290, Theorem 3.1] is not necessary.
\end{abstract}

2000 Mathematics subject classification: primary 20F67.

Keywords and phrases: discreteness criteria, nonelementary, complex hyperbolic spaces, loxodromic.

\section{Introduction}

The discreteness criterion is a very interesting problem in the theory of Möbius groups. This problem has been discussed by many authors and many results can be found in the literature. For instance, in [7], Jørgensen obtained a very useful necessary condition for two-generator Kleinian subgroups of PSL(2, $\mathbb{C})$, which is known as Jørgensen's inequality. As an application, he obtained the following.

THEOREM A. A nonelementary subgroup $G$ of $M\left(\overline{\mathbb{R}}^{2}\right)$ is discrete if and only if each two-generator subgroup of $G$ is discrete.

In [12] Tukia and Wang extended this result as follows.

THEOREM B. Let $G \subset \operatorname{PSL}(2, \mathbb{C})$ be nonelementary. If $G$ contains an elliptic element of order at least 3 , then $G$ is discrete if and only if each nonelementary subgroup generated by two elliptic elements of $G$ is discrete.

They also left an open problem: suppose that $G \subset \operatorname{PSL}(2, \mathbb{C})$ is nonelementary and contains parabolic elements and elliptic elements. Then $G$ is discrete if and only if every subgroup generated by a parabolic and an elliptic element is discrete.

The research was partly supported by Program for NCET (No. 04-0783), NSFs of China (No. 10771059) and Hunan (No. 05JJ10001).

(C) 2010 Australian Mathematical Publishing Association Inc. 0004-9727/2010 \$16.00 
In [15] Yang gave this problem a positive answer. He proved that the following theorem.

Theorem C. Let $G \subset \operatorname{PSL}(2, \mathbb{C})$ be nonelementary. If $G$ contains elliptic elements and parabolic elements, then $G$ is discrete if and only if every subgroup generated by a parabolic and an elliptic element is discrete.

In [16] Yang found analogues of Theorems B and C in $M\left(\overline{\mathbb{R}}^{n}\right)$. His results are as follows.

THEOREM D. Let $G$ be an $n$-dimensional subgroup of $M\left(\overline{\mathbb{R}}^{n}\right)$ containing parabolic and elliptic elements. Then $G$ is discrete if and only if for each parabolic $f$ and elliptic $g$ in $G$, the subgroup $\langle f, g\rangle$ is discrete.

THEOREM E. Let $G$ be an n-dimensional subgroup of $M\left(\overline{\mathbb{R}}^{n}\right)$ containing an elliptic element of order at least 3. Then $G$ is discrete if and only if for each pair of elliptic elements $f$ and $g$ in $G$ the nonelementary subgroup $\langle f, g\rangle$ is discrete.

Here ' $G$ is $n$-dimensional' means that $G$ is nonelementary and does not have a $G$ invariant proper hyperbolic subspace. See [10, 14] for further generalizations of Theorem $\mathrm{A}$ in $M\left(\overline{\mathbb{R}}^{n}\right)$.

For a subgroup $G \subset \mathrm{PU}(1, n)$, let

$$
\begin{aligned}
\mathcal{E}(G) & =\{f \in G \mid f \text { is elliptic }\}, \\
\mathcal{H}(G) & =\{f \in G \mid f \text { is loxodromic }\}, \\
\mathcal{P}(G) & =\{f \in G \mid f \text { is parabolic }\}
\end{aligned}
$$

and

$$
G_{f}=\{g \in G \mid g \text { is conjugate to } f \text { and }\langle f, g\rangle \text { is nonelementary }\} .
$$

Because of the close connection between real and complex hyperbolic spaces it is worthwhile to investigate results analogous to Theorems A-E in the setting of complex hyperbolic space. There is some research in this direction: see $[3,5,8,9]$.

A subgroup $G$ of $\mathrm{PU}(1, n)$ is said to satisfy Condition $C$ if $G$ contains no sequence $\left\{f_{i}\right\}$ such that each $f_{i}$ is parabolic and $f_{i} \rightarrow I$ as $i \rightarrow \infty$. Here $G \subset \mathrm{PU}(1, n)$ is nonelementary if $G$ contains two nonelliptic elements with distinct fixed points. See Section 2 for the definition of $\phi(G)$.

Recently, in [2], Cao proved the following theorems.

Theorem F. Let $G \subset \mathrm{PU}(1, n)$ be nonelementary. Then $G$ is discrete if and only if $\operatorname{ker}(\phi)$ is discrete and each nonelementary subgroup generated by two elements of $\phi(G)_{f}$ is discrete, where $f \in \mathcal{H}(\phi(G))$. If $\mathcal{P}(\phi(G)) \neq \emptyset$, then $G$ is discrete if and only if $\operatorname{ker}(\phi)$ is discrete and each nonelementary subgroup generated by two elements of $\phi(G)_{f}$ is discrete, where $f \in \mathcal{P}(\phi(G))$.

Theorem G. Let $G \subset \mathrm{PU}(1, n)$ be nonelementary and satisfy Condition $C$. If $\mathcal{E}(G) \neq \emptyset$ and it contains an element of order at least 3 , then $G$ is discrete if and only 
if $\operatorname{ker}(\phi)$ is discrete and each nonelementary subgroup generated by two elements in $\mathcal{E}(G)$ is discrete.

The main results of this paper are as follows.

THEOREM 1.1. Let $G \subset \mathrm{PU}(1, n)$ be nonelementary. If $\mathcal{E}(G)$ contains an element of order at least 3, then $G$ is discrete if and only if $\operatorname{ker}(\phi)$ is discrete and every nonelementary subgroup generated by two elements in $\mathcal{E}(G)$ is discrete.

THEOREM 1.2. Let $G \subset \mathrm{PU}(1, n)$ be nonelementary. If $\mathcal{E}(G) \neq \emptyset$ and $\mathcal{P}(G) \neq \emptyset$ then $G$ is discrete if and only if $\operatorname{ker}(\phi)$ is discrete and every subgroup $\langle f, g\rangle$ is discrete, where $f \in \mathcal{P}(G)$ and $g \in \mathcal{E}(G)$.

THEOREM 1.3. Let $G \subset \mathrm{PU}(1, n)$ be nonelementary and $\mathcal{E}(G) \neq \emptyset$. Then $G$ is discrete if and only if $\operatorname{ker}(\phi)$ is discrete and for every subgroup $\langle f, g\rangle$ is discrete, where $f \in \mathcal{H}(G)$ and $g \in \mathcal{E}(G)$.

THEOREM 1.4. $G \subset \mathrm{PU}(1, n)$ be nonelementary and $\mathcal{P}(G) \neq \emptyset$. Then $G$ is discrete if and only if $\operatorname{ker}(\phi)$ is discrete and for every subgroup $\langle f, g\rangle$ is discrete, where $f \in \mathcal{P}(G)$ and $g \in \mathcal{H}(G)$.

REMARK 1.5. Theorem 1.1 shows that Condition $C$ in [2, Theorem 1.4] is removable. Theorem 1.3 shows that the Parabolic Condition assumption in [10, Theorem 3.1] is not necessary in complex hyperbolic spaces. Theorems 1.2 and 1.4 are analogues of [10, Theorems 3.2 and 3.3] in the setting of $\operatorname{PU}(1, n)$.

REMARK 1.6. For a nonelementary subgroup $G \subset M\left(\overline{\mathbb{R}}^{n}\right), G$ is $n$-dimensional if and only if $\operatorname{ker}(\phi)=\{I\}$. Hence in the setting of $M\left(\overline{\mathbb{R}}^{n}\right)$, Theorems 1.1 and 1.2 are generalizations of Theorems D and E. Also, Theorem 1.2 can be regarded as an affirmative answer to Tukia and Wang's open problem in the setting of $\mathrm{PU}(1, n)$.

Refer to $[1-6,8,11]$ for more details on the properties of subgroups of $\mathrm{PU}(1, n)$.

\section{Preliminaries}

Throughout this paper, we adopt the definitions and notation of $[2,4]$, such as $\mathbb{H}_{\mathbb{C}}^{n}$, PU $(1, n)$, discrete group, limit set $L(G)$, and so on. We denote by $M(G)$ the smallest invariant totally geodesic submanifold of $G$ and $\phi(g)$ is the restriction of $g$ to $M(G)$ for all $g \in G$; that is,

$$
\phi(g)=\left.g\right|_{M(G)}, \quad \phi(G)=\left\{\left.g\right|_{M(G)} \mid g \in G\right\} .
$$

According to $[4,6]$, we know that, by conjugation,

$$
M(G)=\mathbb{H}_{\mathbb{C}}^{k} \text { or } \mathbb{H}_{\mathbb{R}}^{l},
$$

where $k$ and $l$ are positive integers and $k, l \leq n$. It is obvious that if $G \subset \mathrm{PU}(1, n)$ and $M(G)=\mathbb{H}_{\mathbb{C}}^{k}$ (respectively $\left.\mathbb{H}_{\mathbb{R}}^{l}\right)$, then for any $g \in G, \phi(g)$ is an element of $\operatorname{PU}(1, k)$ (respectively $\mathrm{PO}(1, l)$ ). 
In order to prove our main results, we need the following lemmas.

LEMMA 2.1 [2]. Let $\phi$ be as in (2.1). Then $G$ is discrete if and only if $\operatorname{ker}(\phi)$ is discrete and $\phi(G)$ is discrete.

LEMMA 2.2 [4]. Let $G \subset \mathrm{SU}(1, m)$ be nonelementary. If $G$ does not leave invariant a proper totally geodesic submanifold of $\mathbb{H}_{\mathbb{C}}^{m}$, then $G$ is either dense or discrete. Moreover, if the identity is not an accumulation point of the elliptic elements in $G$, then $G$ is discrete.

Lemma $2.3[2,5]$. Suppose that two elements $f$ and $g$ in $\mathrm{PU}(1, n)$ generate a discrete and nonelementary group.

(1) If $f$ is parabolic or loxodromic, then

$$
\max \{N(f), N([f, g])\} \geq 2-\sqrt{3},
$$

where $[f, g]=f g f^{-1} g^{-1}$ is the commutator of $f$ and $g, N(f)=\|f-I\|$ and $\|\cdot\|$ means the Frobenius matrix norm such that $\|Q\|=\left[\operatorname{tr}\left(Q Q^{*}\right)\right]^{1 / 2}$ for any matrix $Q$.

(2) If $f$ is elliptic, then

$$
\max \left\{N(f), N\left(\left[f, g^{q}\right]\right) \mid q=1,2,3, \ldots, n+1\right\} \geq 2-\sqrt{3} .
$$

LEMMA 2.4. Let $g$ be an element of $G \subset \mathrm{PU}(1, n)$ and $M(G)=\mathbb{H}_{\mathbb{C}}^{k}$ (respectively $\mathbb{H}_{\mathbb{R}}^{l}$ ). Then:

(1) $g$ is loxodromic if and only if $\phi(g)$ is a loxodromic element in $\mathrm{PU}(1, k)$ (respectively $\mathrm{PO}(1, l))$;

(2) $g$ is parabolic if and only if $\phi(g)$ is a parabolic element in $\mathrm{PU}(1, k)$ (respectively $\mathrm{PO}(1, l))$;

(3) $g$ is elliptic if and only if $\phi(g)$ is an elliptic or identity element in $\mathrm{PU}(1, k)$ (respectively $\mathrm{PO}(1, l)$ ).

PROOF. This is obvious from [4, p. 77].

LEMMA 2.5. Let $G \subset \mathrm{SU}(1, m)$ (respectively $\mathrm{SO}(1, m)$ ) be nonelementary. If $G$ is not discrete and does not leave invariant a proper totally geodesic submanifold, then there exists a sequence $\left\{f_{i}\right\} \subset G$ such that each $f_{i}$ is loxodromic and $f_{i} \rightarrow I$ as $i \rightarrow \infty$.

Proof. By [4, Theorem 4.4.2 and Corollary 4.5.1], we know that $G$ is dense in $\mathrm{SU}(1, m)$ (respectively $\mathrm{SO}(1, m)$ ), so the closure of $G$ is $\bar{G}=\mathrm{SU}(1, m)$ (respectively $\mathrm{SO}(1, m))$. This implies that there exists a sequence $\left\{g_{i}\right\} \subset \bar{G}$ such that each $g_{i}$ is loxodromic and $g_{i} \rightarrow I$ as $i \rightarrow \infty$.

As the loxodromic elements of $\mathrm{SU}(1, m)$ (respectively $\mathrm{SO}(1, m)$ ) form an open set in the closure $\bar{G}$ of $G$, there must exist a sequence $\left\{f_{i}\right\} \subset G$ such that each $f_{i}$ is loxodromic and $f_{i} \rightarrow I$ as $i \rightarrow \infty$. 
LEMMA 2.6. Let $G$ be an m-dimensional subgroup of $\mathrm{SO}(1, m)$ such that $\mathcal{E}(G) \neq \emptyset$. Then $G$ is discrete if and only if each nonelementary subgroup $\langle f, g\rangle$ is discrete, where $f \in \mathcal{H}(G)$ and $g \in \mathcal{E}(G)$.

PROOF. The necessity is obvious. For the converse, we suppose, to the contrary, that $G$ is not discrete. By Lemma 2.5, we know that there exists a sequence $\left\{f_{i}\right\} \subset G$ such that each $f_{i}$ is loxodromic and $f_{i} \rightarrow I$ as $i \rightarrow \infty$.

Suppose that $g$ is an elliptic element in $G$ such that $g^{2} \neq I$. If, for large enough $i$, the set

$$
S=\left\{\operatorname{fix}\left(f_{i}\right), \operatorname{fix}\left(f_{i+1}\right), \operatorname{fix}\left(f_{i+2}\right), \ldots\right\}
$$

can span the boundary of $M(G)$, then Lemma 2.3 implies that the subgroups $\left\langle g, f_{i}\right\rangle$, $\left\langle g, f_{i+1}\right\rangle,\left\langle g, f_{i+2}\right\rangle, \ldots$ are all discrete and elementary. It is obvious that $g=I$. This is a contradiction.

If, for large enough $i$, the set $S=\left\{f i x\left(f_{i}\right)\right.$, fix $\left(f_{i+1}\right)$, fix $\left.\left(f_{i+2}\right), \ldots\right\}$ cannot span the boundary of $M(G)$, then we could choose a loxodromic element $h$ such that fix $(h) \cap S=\emptyset$. Thus we could find an integer $N$ such that $\operatorname{fix}\left(h^{N} g h^{-N}\right) \cap S=\emptyset$. For sufficiently large $i$,

$$
N\left(f_{i}\right)+N\left(\left[h^{N} g h^{-N}, f_{i}\right]\right)<2-\sqrt{3} .
$$

By Lemma 2.3, we know that the subgroups $\left\langle h^{N} g h^{-N}, f_{i}\right\rangle$ are discrete and elementary. As fix $\left(h^{N} g h^{-N}\right) \cap S=\emptyset$, we again reach a contradiction.

REMARK 2.7. Lemma 2.6 implies that the parabolic condition hypothesis in [10, Theorem 3.1] is not necessary.

\section{Proofs of the theorems}

Proof of TheOrem 1.1. The necessity is obvious. For the converse, we suppose, to the contrary, that $G$ is not discrete. By Lemma 2.1, we know that $\phi(G)$ is not discrete. As $G$ is nonelementary, we may assume that the smallest totally geodesic submanifold $M(G)$ is either $\mathbb{H}_{\mathbb{C}}^{k}$ or $\mathbb{H}_{\mathbb{R}}^{l}$. We divide the proof into two cases.

Case I. $\quad M(G)=\mathbb{H}_{\mathbb{C}}^{m}$. By Lemma 2.2, we know that there exists a sequence $\left\{g_{m+1, i}\right\} \subset \phi(G)$ such that each $g_{m+1, i}$ is elliptic and $g_{m+1, i} \rightarrow I$ as $i \rightarrow \infty$. It is obvious from Lemma 2.4 and [4, p. 77] that there must exist a sequence $\left\{g_{i}\right\} \subset G$ such that each $g_{i}$ is elliptic and $g_{i} \rightarrow I$ as $i \rightarrow \infty$. This violates [2, Lemma 2.7].

Case II. $\quad M(G)=\mathbb{H}_{\mathbb{R}}^{l}$. By Lemma 2.4, $\phi(G)$ is a subgroup of $\mathrm{PO}(1, l)$. As $G$ is nonelementary and $M(G)=H_{\mathbb{R}}^{l}$. Then [4, Corollary 4.5.1] yields that $\phi(G)$ is dense in $\mathrm{SO}(1, l)$. So the set of all orientation-preserving elements in $\mathrm{SO}^{+}(1, l)$ is dense (see [13, Theorem 3.1]). Theorem E and Lemma 2.4 imply that this is a contradiction.

This completes the proof of Theorem 1.1.

Proof of TheOREM 1.2. The necessity is obvious. For the converse, we suppose, to the contrary, that $G$ is not discrete. As in the proof of Theorem 1.1 we divide the proof into two cases. 
Case I. $\quad M(G)=\mathbb{H}_{\mathbb{C}}^{m}$. According to Case $\mathrm{I}$ in the proof of Theorem 1.1, we know that there exists a sequence $\left\{f_{i}\right\} \subset G$ such that each $f_{i}$ is elliptic and $f_{i} \rightarrow I$ as $i \rightarrow \infty$. As $G$ is nonelementary and $\mathcal{P}(G) \neq \emptyset$ there exist finite many parabolic elements $g_{1}, \ldots, g_{s}$ in $G$ such that fix $\left(g_{1}\right), \ldots$, fix $\left(g_{s}\right)$ span the boundary of $M(G)$. Then, for large enough $i$,

$$
N\left(f_{i}\right)+\sum_{q=1}^{n+1} N\left(\left[f_{i}, g_{j}^{q}\right]\right)<2-\sqrt{3},
$$

where $j=1,2, \ldots, s$. So for large enough $i$, the subgroup $\left\langle f_{i}, g_{s}\right\rangle$ is elementary, and hence $\left.f_{i}\right|_{M(G)}=I$. From the definition of $\operatorname{ker}(\phi)$, we know that $f_{i} \in \operatorname{ker}(\phi)$ for sufficient large $i$. This is a contradiction, since $\operatorname{ker}(\phi)$ is discrete.

Case II. $\quad M(G)=\mathbb{H}_{\mathbb{R}}^{l}$. According to Lemma 2.4 and Theorem D, it is easy to see that $\phi(G)$ is discrete. By Lemma 2.1, we know that $G$ is discrete. This is also a contradiction and completes the proof of Theorem 1.2.

Proof of Theorem 1.3. The necessity is obvious. For the converse, we suppose, to the contrary, that $G$ is not discrete. By Lemma 2.6 and reasoning as in the proof of Theorem 1.2, it is easy to get a contradiction.

Proof of TheOrem 1.4. The necessity is obvious. For the converse, we suppose that $G$ is not discrete. Then there exists a sequence $\left\{f_{i}\right\} \subset G$ such that $f_{i} \rightarrow I$ as $i \rightarrow \infty$. As $\operatorname{ker} \phi(G)$ is discrete, there exists a parabolic element $g$ of $G$ such that $\operatorname{fix}\left(f_{i}\right) \cap \operatorname{fix}(g)=\emptyset$. Then $g^{k} f_{i}$ is loxodromic when $k$ is large enough. Lemma 2.3 implies that the subgroup $\left\langle f_{i}, g\right\rangle=\left\langle g, g^{k} f_{i}\right\rangle$ is discrete and elementary. This is the desired contradiction.

\section{Acknowledgements}

Part of this work was finished while the author was visiting the University of Durham. He would like to thank Professor John R. Parker for the invitation and the Mathematics Department for their hospitality. Also, he heartily thanks the referee for a careful reading of this paper as well as some useful comments and suggestions.

\section{References}

[1] A. Basmajian and R. Miner, 'Discrete groups of complex hyperbolic motion', Invent. Math. 131 (1998), 85-136.

[2] W. Cao, 'Discrete and dense subgroups acting on complex hyperbolic space', Bull. Aust. Math. Soc. 78 (2008), 211-224.

[3] W. Cao and X. Wang, 'Discreteness criteria and algebraic convergence theorem for subgroups in PU (1, n; C)', Proc. Japan Acad. 82 (2006), 49-52.

[4] S. S. Chen and L. Greenberg, Hyperbolic Spaces: Constributions to Analysis (Academic Press, New York, 1974), pp. 49-87.

[5] B. Dai, A. Fang and B. Nai, 'Discreteness criteria for subgroups in complex hyperbolic space', Proc. Japan Acad. 77 (2001), 168-172. 
[6] W. M. Goldman, Complex Hyperbolic Geometry (Oxford University Press, New York, 1999).

[7] T. Jørgensen, 'On discrete groups of Möbius transformation', Amer. J. Math. 98 (1976), 739-749.

[8] S. Kamiya, 'Notes on elements of $U(1, n: \mathbb{C})$ ', Hiroshima Math. J. 21 (1991), 23-45.

[9] S. Kamiya, 'Chordal and matrix norms of unitary transformations', First Korean-Japanese Colloquium on Finite or Infinite Dimensional Complex Analysis (1993), 121-125.

[10] L. Li and X. Wang, 'Discreteness criteria for Möbius groups acting on $\overline{\mathbb{R}}^{n} \mathrm{II}$ ', Bull. Aust. Math. Soc. 80 (2009), 275-290.

[11] J. R. Parker, 'Uniform discreteness and Heisenberg transformation', Math. Z. 225 (1997), 485-505.

[12] P. Tukia and X. Wang, 'Discreteness of subgroup of $S L(2, \mathbb{C})$ containing elliptic elements', Math. Scand. 91 (2004), 214-220.

[13] X. Wang, 'Dense subgroups of $n$-dimensional Möbius groups', Math. Z. 243 (2003), 643-651.

[14] X. Wang, L. Li and W. Cao, 'Discreteness criteria for Möbius groups acting on $\overline{\mathbb{R}}^{n}$, Israel J. Math. 150 (2005), 357-368.

[15] S. Yang, 'On the discreteness in $S L(2, \mathbb{C})$ ', Math. Z. 255 (2007), 227-230.

[16] S. Yang, 'Discreteness criteria for isometric groups of real and complex hyperbolic space', Indiana Univ. Math. J. 58 (2009), 1443-1456.

XI FU, Department of Mathematics, Hunan Normal University, Changsha, Hunan 410081, PR China

e-mail: fuxi1000@yahoo.com.cn 